\title{
CALCULATION OF THE PRESSURE ON THE VALVES OF A SLUICE
}

\author{
Tran Gia Lich - Le KIM LUaT - HaN QUOC Trinh \\ Institute of Mathematics, Hanoi, Vietnam
}

\begin{abstract}
This paper is devoted to a numerical method for calculating the pressure on the vertical two-dimensional valve basing on Navier-Stokes equations. Numerical solutions at interior points are established by splitting Navier-Stokes unsteady two-dimensional equations into two unsteady one-dimensional equations. An implicit scheme is obtained and the solution for these equations is established by the double sweep method. The values at the boundary points are calculated by the method of characteristics.
\end{abstract}

This algorithm is applied to the concrete case presented at the end of this paper

\section{Introduction}

It is very difficult to calculate the pressure fields, especially, in the case of solid boundary. Much attention has been paid to this problem. The aim of this paper is to present a numerical method for calculating the pressure on the vertical two-dimensional valve in hydraulic engineering.

It is well known that the Navier-Stock equations for viscous incompressible fluid flows have the dimensional form as following:

$$
\begin{aligned}
& \frac{\partial \mathbf{V}}{\partial t}+(\mathbf{V} \cdot \nabla) \mathbf{V}=-\frac{1}{\rho} \nabla P+\nu \Delta \mathbf{V}+\mathbf{F} \\
& \nabla \cdot \mathbf{V}=0
\end{aligned}
$$

where $\mathbf{V}$ is velocity vector, $P$ - pressure, $\mathbf{F}$ - external force, $\rho$ - density, $\nu$ - kinematics viscosity. Let take $\rho=1$.

It is difficult to find directly numerical solutions of equations (1.1). To avoid it, the artificial compression component is added to the continuity equation (see $[1,2]$ ), and we obtain a modification for the Navier-Stokes equations as follows:

$$
\begin{aligned}
& \frac{\partial \mathbf{V}}{\partial t}+(\mathbf{V} \cdot \nabla) \mathbf{V}=-\nabla P+\nu \Delta \mathbf{V}+\mathbf{F} \\
& \frac{\partial\left(P+\frac{\mathbf{V}^{2}}{4}\right)}{\partial t}+\nabla \cdot \mathbf{V}=0 .
\end{aligned}
$$

We suppose that either the channel has large enough width (in $O y$-direction) or the velocity of fluid flow changes slowly in $O y$-direction, then we can rewrite equations (1.2) in the vertical two-dimensional equations of fluid flow (in $(x, z)$-direction).

The authors of $[5]$ have considered the vertical two-dimensional fluid flow, the governing equations of which have following forms:

$$
\begin{aligned}
& \frac{\partial u}{\partial t}+u \frac{\partial u}{\partial x}+w \frac{\partial u}{\partial z}+\frac{\partial p}{\partial x}=i g+\nu \Delta u \\
& \frac{\partial w}{\partial t}+u \frac{\partial w}{\partial x}+w \frac{\partial w}{\partial z}+\frac{\partial p}{\partial z}=-\sqrt{1-i^{2}} g+\nu \Delta w
\end{aligned}
$$




$$
\frac{\partial u}{\partial x}+\frac{\partial w}{\partial z}=0
$$

where $g$ is the acceleration of gravity, $i=\sin \xi$, and $\xi$ is the angle of inclination between the gravity and the $O z$-axis.

The first component on the right of two first equations (1.3) represents projections of gravity onto coordinate axes.

According to the idea mentioned above, the continuity equation (1.4) can be changed into:

$$
\frac{\partial q}{\partial t}+\frac{\partial u}{\partial x}+\frac{\partial w}{\partial z}=0
$$

where $q=p+\frac{v^{2}}{4}$.

The equations (1.3), (1.5) are solved by the splitting method, particularly, we solve each one-dimensional equation in $x, z$ - directions respectively, as follows (see [1]):

$$
\begin{aligned}
& \frac{1}{2} \frac{\partial u}{\partial t}+\frac{\partial q_{1}}{\partial x}=\nu \frac{\partial^{2} u}{\partial x^{2}}+i g \\
& \frac{1}{2} \frac{\partial w}{\partial t}+u \frac{\partial w}{\partial x}=\nu \frac{\partial^{2} w}{\partial x^{2}} \\
& \frac{1}{2} \frac{\partial q_{1}}{\partial t}+\frac{\partial u}{\partial x}=0 \\
& \frac{1}{2} \frac{\partial u}{\partial t}+w \frac{\partial u}{\partial z}=\nu \frac{\partial^{2} u}{\partial z^{2}} \\
& \frac{1}{2} \frac{\partial w}{\partial t}+\frac{\partial q_{2}}{\partial z}=\nu \frac{\partial^{2} w}{\partial z^{2}}-\sqrt{1-i^{2}} g \\
& \frac{1}{2} \frac{\partial q_{2}}{\partial t}+\frac{\partial w}{\partial z}=0
\end{aligned}
$$

where

$$
q_{1}=p+\frac{u^{2}}{2}, \quad q_{2}=p+\frac{w^{2}}{2} \quad \text { and } \quad q=\frac{1}{2}\left(q_{1}+q_{2}\right)
$$

\section{Algorithm}

The main idea solving equations (1.6), (1.7) is as follows:

Firstly, under the given initial conditions, we find numerical solutions of equations (1.6) along the line $z=$ const at the first half-time-step, then, we use the results obtained from this stage as initial conditions to solve equations (1.7) along the line $x=$ const at the other half-time-step. The obtained results are considered as the numerical solutions of equations (1.3), (1.5) in the whole time step.

In order to find the numerical solution of equations (1.6), (1.7), we have to calculate the numerical values at each point of the considered region, i.e. at the interior points and at the boundary points.

\section{A. At the interior points of calculated region}

In order to determine $u, w$ and $p$ values (as well as $u, w, q_{1}, q_{2}$ ) at the interior points, we use implicit finite-difference scheme to solve equations (1.6), (1.7). The following finite-difference equations are obtained 


$$
\begin{aligned}
& \frac{u^{n+\frac{1}{2}}-u^{n}}{d t}+\frac{\Delta_{1}}{d x}\left(\alpha q_{1}^{n+\frac{1}{2}}+\beta q_{1}^{n}\right)=\nu \frac{\Delta_{1} \Delta_{-1}}{(d x)^{2}}\left[\alpha u^{n+\frac{1}{2}}+\beta u^{n}\right]+i g, \\
& \frac{w^{n+\frac{1}{2}}-w^{n}}{d t}+\left(\alpha u^{n+\frac{1}{2}}+\beta u^{n}\right) \frac{\Delta}{d x}\left[\alpha w^{n+\frac{1}{2}}+\beta w^{n}\right]=\nu \frac{\Delta_{1} \Delta_{-1}}{(d x)^{2}}\left[\alpha w^{n+\frac{1}{3}}+\beta w^{n}\right], \\
& \frac{q_{1}^{n+\frac{1}{2}}-q_{1}^{n}}{d t}+\frac{\Delta_{-1}}{d x}\left[\alpha u^{n+\frac{1}{2}}+\beta u^{n}\right]=0 \\
& \frac{u^{n+1}-u^{n+\frac{1}{2}}}{d t}+\left(\alpha w^{n+1}+\beta w^{n+\frac{1}{2}}\right) \frac{\delta}{d z}\left(\alpha u^{n+1}+\beta u^{n+\frac{1}{2}}\right)=\nu \frac{\Delta_{2} \Delta_{-2}}{(d z)^{2}}\left[\alpha u^{n+1}+\beta u^{n+\frac{1}{2}}\right], \\
& \frac{w^{n+1}-w^{n+\frac{1}{2}}}{d t}+\frac{\Delta_{2}}{d z}\left(\alpha q_{2}^{n+1}+\beta q_{2}^{n+\frac{1}{2}}\right)=\nu \frac{\Delta_{2} \Delta_{-2}}{(d z)^{2}}\left(\alpha w^{n+1}+\beta w^{n+\frac{1}{2}}\right)-\sqrt{1-i^{2}} g, \\
& \frac{q_{2}^{n+1}-q_{2}^{n+\frac{1}{2}}}{d t}+\frac{\Delta_{-2}}{d z}\left[\alpha w^{n+1}+\beta w^{n+\frac{1}{2}}\right]=0 .
\end{aligned}
$$

where $d x, d z$ are the spatial steps in $x$ - and $z$-direction respectively, $d t$ is the time-step, $\alpha+\beta=1$, $0 \leq \alpha, \beta \leq 1$,

$$
\begin{aligned}
& \Delta_{ \pm i}(i=1,2) \text { are difference operators with } \\
& i=1 \text { : in } x \text {-direction } \\
& i=2: \text { in } z \text {-direction } \\
& \Delta_{1} \varphi=\varphi_{m+1, k}-\varphi_{m, k} ; \quad \Delta_{2} \varphi=\varphi_{m, k+1}-\varphi_{m, k} \\
& \Delta_{-1} \varphi=\varphi_{m, k}-\varphi_{m-1, k} ; \Delta_{-2} \varphi=\varphi_{m, k}-\varphi_{m, k-1} \text {. }
\end{aligned}
$$

Now the Courant-Levi stability condition (see [6]) can be applied:

$$
\begin{aligned}
& \Delta=\Delta_{-1} \text { if } \alpha u^{n+\frac{1}{2}}+\beta u^{n} \geq 0 \\
& \Delta=\Delta_{1} \text { if } \alpha u^{n+\frac{1}{2}}+\beta u^{n}<0 \\
& \delta=\Delta_{-2} \text { if } \alpha w^{n+1}+\beta w^{n+\frac{1}{2}} \geq 0 \\
& \delta=\Delta_{2} \text { if } \alpha w^{n+1}+\beta w^{n}<0
\end{aligned}
$$

or one can choose $\Delta, \delta$ as central differences.

For the sake of brevity, only the algorithm for the case of central differences $\Delta$ and $\delta$ is described. The full algorithm will be realized in FORTRAN 77.

It is very easy to check the differential coefficients (2.1), (2.2) having first order approximations for $d t, d x$ and $d z$, except for the case $\alpha=\beta=\frac{1}{2}$, we have second-order approximations for $d x, d z$. The scheme is explicit when $\alpha=0, \beta=1$ and implicit otherwise.

It is easy to see that the implicit finite-difference equations $(2.1),(2.2)$ can be drawn double sweep form at grid points in $x, z$-directions.

a) The implicit finite-difference equations (2.1) along $x$-direction for $u_{m, k}^{n+\frac{1}{2}}, w_{m, k}^{n+\frac{1}{2}}$ and $q_{1, m, k}^{n+\frac{1}{2}}$

$\left.a_{1}\right)$

$$
\begin{aligned}
& u_{m, k}^{n+\frac{1}{2}}=L_{m} u_{m+1, k}^{n+\frac{1}{2}}+K_{m} \\
& \left(M_{k}^{0}+1 \leq m \leq M_{k}^{1}-1\right)
\end{aligned}
$$


where

$$
\begin{aligned}
a_{m} & =-\frac{\alpha}{(d x)^{2}}(d t . \alpha+\nu), \\
b_{m} & =\frac{1}{d t}+\frac{2 . \alpha}{(d x)^{2}}(d t . \alpha+\nu) ; \quad c_{m}=a_{m}, \\
f_{m} & =\frac{1}{d x}\left(-q_{1, m+1, k}^{n}+q_{1, m, k}^{n}\right)+\frac{1-\alpha}{(d x)^{2}}(d t . \alpha+\nu)\left(u_{m+1, k}^{n}-2 u_{m, k}^{n}+u_{m-1, k}^{n}\right)+\frac{1}{d t} u_{m, k}^{n}+i g \\
L_{m} & =-\frac{a_{m}}{b_{m}+c_{m} L_{m-1}} ; \quad K_{m}=\frac{f_{m}-c_{m} K_{m-1}}{b_{m}+c_{m} L_{m-1}} .
\end{aligned}
$$

It is easy verify that $\left|b_{m}\right|>\left|a_{m}\right|+\left|c_{m}\right|+\delta, \delta>0$. Therefore the double sweep method do not accumulate the computional error (see [6]).

Note. $M_{k}^{0}, M_{k}^{1}$ are the numbers of the left boundaries and the right ones respectively, at $K^{-t h}$ row:

$u_{M_{k}^{0}, k}=\varphi_{k}^{1} ; \quad u_{M_{k}^{1}, k}=\psi_{k}^{1} ; K_{M_{k}^{0}}=\varphi_{k}^{1} ; L_{M_{k}^{0}}=0$,

where $\varphi_{k}^{1}, \psi_{k}^{1}$ are the known values at the boundary.

$\left.a_{2}\right)$

$$
\begin{gathered}
q_{1, m, k}^{n+\frac{1}{2}}=q_{1, m, k}^{n}-\frac{d t}{d x}\left(\alpha\left(u_{m, k}^{n+\frac{1}{2}}-u_{m-1, k}^{n+\frac{1}{2}}\right)+(1-\alpha)\left(u_{m, k}^{n}-u_{m-1, k}^{n}\right)\right) \\
\left(M_{k}^{0}+1 \leq m \leq M_{k}^{1}-1\right)
\end{gathered}
$$

$\left.a_{3}\right)$

$$
\begin{gathered}
w_{m, k}^{n+\frac{1}{2}}=L_{m} W_{m+1, k}^{n+\frac{1}{2}}+K_{m} \\
\left(M_{k}^{0}+1 \leq m \leq M_{k}^{1}-1\right)
\end{gathered}
$$

where

$$
\begin{aligned}
a_{m}= & \frac{\alpha\left(\alpha u_{m, k}^{n+\frac{1}{2}}+(1-\alpha) u_{m, k}^{n}\right)}{2 d x}-\frac{\nu \alpha}{(d x)^{2}}, \\
b_{m}= & \frac{1}{d t}+\frac{2 \nu \alpha}{(d x)^{2}} ; \quad c_{m}=-a_{m}-2 \frac{\nu \alpha}{(d x)^{2}}, \\
f_{m}= & \frac{w_{m, k}^{n}}{d t}-\frac{\left(\alpha u_{m, k}^{n+\frac{1}{2}}+(1-\alpha) u_{m, k}^{n}\right)(1-\alpha)\left(w_{m+1, k}^{n}-w_{m-1, k}^{n}\right)}{2 d x} \\
& +\frac{\nu(1-\alpha)}{(d x)^{2}}\left(w_{m+1, k}^{n}-2 w_{m, k}^{n}+w_{m-1, k}^{n}\right) \\
L_{m}= & -\frac{a_{m}}{b_{m}+c_{m} L_{m-1}} ; \quad K_{m}=\frac{f_{m}-c_{m} K_{m-1}}{b_{m}+c_{m} L_{m-1}} \\
W_{M_{k}^{0}, k}^{n+\frac{1}{2}}= & \varphi_{k} ; \quad W_{M_{k}^{1}, k}^{n+\frac{1}{2}}=\psi_{k} ; \quad K_{M_{k}^{0}}=\varphi_{k} ; \quad L_{M_{k}^{0}}=0
\end{aligned}
$$

where $\varphi_{k}, \psi_{k}$ are the known values at the boundary.

b) The implicit finite-difference equation (2.2) along $z$-direction for $W_{m, k}^{n+1}, q_{2, m, k}^{n+1}$ and $u_{m, k}^{n+1}$

$K_{m}^{1}, K_{m}^{0}$ are the numbers of the upper and lower boundaries at the $m^{-t h}$ column, respectively.

$\left.b_{1}\right)$

$$
\begin{gathered}
W_{m, k}^{n+1}=L_{k} W_{m, k+1}^{n+1}+K_{k} \\
\left(K_{m}^{0}+1 \leq k \leq K_{m}^{1}-1\right)
\end{gathered}
$$


where

$$
\begin{aligned}
a_{k}= & -\frac{\alpha}{(d z)^{2}}(d t \alpha+\nu) \\
b_{k}= & \frac{1}{d t}+\frac{2 \alpha}{(d z)^{2}}(d t \alpha+\nu) ; \quad c_{k}=a_{k} \\
f_{k}= & \frac{1}{d z}\left(-q_{2, m, k+1}^{n+\frac{1}{2}}+q_{2, m, k}^{n+\frac{1}{2}}\right)+\frac{1-\alpha}{(d z)^{2}}(\alpha d t+\nu)\left(W_{m, k+1}^{n+\frac{1}{2}}-2 W_{m, k}^{n+\frac{1}{2}}+W_{m, k-1}^{n+\frac{1}{2}}\right) \\
& +\frac{1}{d t} W_{m, k}^{n+\frac{1}{2}}-\sqrt{1-i^{2}} g \\
L_{k}= & \frac{-a_{k}}{b_{k}+c_{k} L_{k-1}} ; \quad K_{k}=\frac{f_{k}-c_{k} K_{k-1}}{b_{k}+c_{k} L_{k-1}} \\
W_{m, K_{m}^{0}}^{n+1}= & \varphi_{m} ; W_{m, K_{m}^{1}}^{n+1}=\psi_{m} ; K_{K_{m}^{0}}=\varphi_{m} ; \quad L_{K_{m}^{0}}=0 ;
\end{aligned}
$$

$\varphi_{m}, \psi_{m}$ are the known values at the boundary.

It is easy to see that $\left|b_{k}\right|>\left|a_{k}\right|+\left|c_{k}\right|+\delta, \delta>0$.

$\left.b_{2}\right)$

$$
\begin{gathered}
q_{2, m, k}^{n+1}=q_{2, m, k}^{n+\frac{1}{2}}-\frac{d t}{d z}\left[\alpha\left(W_{m, k}^{n+1}-W_{m, k-1}^{n+1}\right)+(1-\alpha)\left(W_{m, k}^{n+\frac{1}{2}}-W_{m, k-1}^{n+\frac{1}{2}}\right)\right] \\
\left(K_{m}^{0}+1 \leq k \leq K_{m}^{1}+1\right)
\end{gathered}
$$

$\left.b_{3}\right)$

$$
\begin{aligned}
& u_{m, k}^{n+1}=L_{k} u_{m, k+1}^{n+1}+K_{k} \\
& \left(K_{m}^{0}+1 \leq k \leq K_{m}^{1}-1\right)
\end{aligned}
$$

where

$$
\begin{aligned}
a_{k}= & \frac{\alpha\left(\alpha W_{m, k}^{n+1}+(1-\alpha) W_{m, k}^{n+\frac{1}{2}}\right.}{2 d z}-\frac{\nu \alpha}{(d z)^{2}} \\
b_{k}= & \frac{1}{d t}+\frac{2 \nu \alpha}{(d z)^{2}} ; \quad c_{k}=-a_{k}-2 \frac{\nu a}{(d z)^{2}} \\
f_{k}= & \frac{u_{m, k}^{n+\frac{1}{2}}}{d t}-\frac{\left(\alpha W_{m, k}^{n+1}+(1-\alpha) W_{m, k}^{n+\frac{1}{2}}\right)(1-\alpha)\left(u_{m, k+1}^{n+\frac{1}{2}}-u_{m, k-1}^{n+\frac{1}{2}}\right)}{2 d z} \\
& +\frac{\nu(1-\alpha)}{(d z)^{2}}\left(u_{m, k+1}^{n+\frac{1}{2}}-2 u_{m, k}^{n+\frac{1}{2}}+u_{m, k-1}^{n+\frac{1}{2}}\right) \\
L_{k}= & -\frac{a_{k}}{b_{k}+c_{k} L_{k-1}} ; \quad K_{k}=\frac{f_{k}-c_{k} K_{k-1}}{b_{k}+c_{k} L_{k-1}} \\
u_{m . K_{m}^{n}}^{n+1}= & \varphi_{m} ; \quad u_{m, K_{m}^{k}}^{n+1}=\psi_{m} ; \quad K_{K_{m}^{0}}=\varphi_{m} ; \quad L_{K_{m}^{0}}=0
\end{aligned}
$$

$\varphi_{m}, \psi_{m}$ are the known values at boundary.

Remark. After finding $u^{n+\frac{1}{2}}, w^{n+\frac{1}{2}}$ and $q_{1}^{n+\frac{1}{3}}$ at the first half-time-step, for the other half-timestep, we have to find $q_{2}$ by using the formula:

$$
q_{2}^{n+\frac{1}{2}}=p^{n+\frac{1}{2}}+\frac{\left(w^{n+\frac{1}{2}}\right)^{2}}{2}
$$

where

$$
p^{n+\frac{1}{2}}=q_{1}^{n+\frac{1}{2}}-\frac{\left(u^{n+\frac{1}{2}}\right)^{2}}{2} .
$$


We shall obtain, $u^{n+1}$ and $q_{2}^{n+1}$, and in order to find out $u^{n+\frac{3}{2}}, w^{n+\frac{3}{2}}$ and $q_{1}^{n+\frac{3}{2}}$, we have to find $q_{1}^{n+1}$ as follows:

$$
q_{1}^{n+1}=p^{n+1}+\frac{\left(u^{n+1}\right)^{2}}{2}
$$

where

$$
p^{n+1}=q_{2}^{n+1}-\frac{\left(w^{n+1}\right)^{2}}{2} .
$$

The above process is repeated until we obtain the results satisfying acceptable accuracy.

\section{B. At the boundary points}

To find $u, w, q_{1}$ and $q_{2}$ at the boundary points, we shall apply the method of characteristics $(\operatorname{see}[3,4])$.

Equations (1.6) are written in vector form as follows:

$$
\frac{\partial \mathbf{V}_{1}}{\partial t}+\mathbf{A} \frac{\partial \mathbf{V}_{1}}{\partial x}=\mathbf{F}^{x}
$$

where

$$
\mathbf{V}_{1}=\left(\begin{array}{c}
u \\
w \\
q_{1}
\end{array}\right) ; \quad \mathbf{A}=\left(\begin{array}{ccc}
0 & 0 & 2 \\
0 & 2 u & 0 \\
2 & 0 & 0
\end{array}\right) ; \quad \mathbb{F}^{x}=\left(\begin{array}{c}
f_{1}^{x} \\
f_{2}^{x} \\
f_{3}^{x}
\end{array}\right)=\left(\begin{array}{c}
2 \nu \frac{\partial^{2} u}{\partial x^{2}}+2 i g \\
2 \nu \frac{\partial^{2} w}{\partial x^{2}}
\end{array}\right)
$$

and $\frac{\partial p}{\partial t}$ or $\frac{\partial}{\partial t}\left(p+\frac{u^{2}}{2}\right)$ can be chosen as $f_{3}^{x}$ at the previous (n-th) time-step.

The vector of left eigenvalues of the matrix $\mathbf{A}$ is $\lambda^{x}=\left(\lambda_{1}^{x}, \lambda_{2}^{x}, \lambda_{3}^{x}\right)=(2,-2,2 u)$ and the corresponding matrix of left eigenvectors is

$$
\mathbf{\Omega}_{A}=\left(\begin{array}{ccc}
1 & 0 & 1 \\
1 & 0 & -1 \\
0 & 1 & 0
\end{array}\right) \quad \text { and } \mathbb{\Omega}_{A}^{-1}=\frac{1}{2}\left(\begin{array}{ccc}
1 & 1 & 0 \\
0 & 0 & 2 \\
1 & -1 & 0
\end{array}\right)
$$

Since $\lambda_{i}^{x}(i=1,2,3)$ are real different from each other, the equations (2.9) is a hyperbolic system. After some equivalent transformations for system (2.9), we obtain the formula:

$$
\mathbb{R}_{A}^{-1}\left\|\vec{\tau}_{i}\left(\frac{d \mathbf{V}_{1}}{d t}\right)_{x_{i}}\right\|=\mathbf{F}^{x}
$$

where

$$
\mathbf{n}_{A}=\left(\begin{array}{l}
\vec{\tau}_{1} \\
\vec{\tau}_{2} \\
\vec{\tau}_{3}
\end{array}\right),\left\|\vec{\tau}_{i}\left(\frac{d \mathbf{V}_{1}}{d t}\right)_{x_{i}}\right\|=\left(\begin{array}{l}
\vec{\tau}_{1}\left(\frac{d \mathbf{V}_{1}}{d t}\right)_{x_{1}} \\
\vec{\tau}_{2}\left(\frac{d \mathbf{V}_{1}}{d t}\right)_{x_{2}} \\
\vec{\tau}_{3}\left(\frac{d \mathbf{V}_{1}}{d t}\right)_{x_{3}}
\end{array}\right)
$$

and $\left(\frac{d u}{d t}\right)_{x_{i}}$ is the derivative along the direction of the characteristical line $\frac{d x}{d t}=\lambda_{i}^{x}$, i.e.

$$
\left(\frac{d u}{d t}\right)_{x i}=\frac{\partial u}{\partial t}+\lambda_{i}^{x} \frac{\partial u}{\partial x} ; \quad(i=1,2,3)
$$


This formula is rewritten as follows:

$$
\begin{aligned}
& \left(\frac{d u}{d t}\right)_{x_{1}}+\left(\frac{d q_{1}}{d t}\right)_{x_{1}}+\left(\frac{d u}{d t}\right)_{x_{2}}-\left(\frac{d q_{1}}{d t}\right)_{x_{2}}=2 f_{1}^{x} \\
& \left(\frac{d w}{d t}\right)_{x_{1}}=f_{2}^{x} \\
& \left(\frac{d u}{d t}\right)_{x_{1}}+\left(\frac{d q_{1}}{d t}\right)_{x_{1}}-\left(\frac{d u}{d t}\right)_{x_{1}}+\left(\frac{d q_{1}}{d t}\right)_{x_{1}}=0 .
\end{aligned}
$$

It is necessary to know the values $u, w$ and $p$ at any boundary point, in order that the boundary equations are closed, so except the given boundary conditions, the following complementary equations for each boundary are derived from formula $(2.10)$ as follows:

- At the right boundary:

$$
\left(\frac{d u}{d t}\right)_{x_{1}}+\left(\frac{d \dot{q}_{1}}{d t}\right)_{x_{1}}=f_{1}^{x}
$$

and if $u \geq 0$ we shall have one more equation:

$$
\left(\frac{d w}{d t}\right)_{x_{1}}=f_{2}^{x}
$$

- At the left boundary:

$$
\left(\frac{d u}{d t}\right)_{x_{2}}-\left(\frac{d q_{1}}{d t}\right)_{x_{3}}=f_{1}^{x}
$$

and if $u \leq 0$ we shall have one more equation (2.12).

By the same way, we derive from (1.7) the following equation:

$$
\frac{\partial \mathbf{V}_{2}}{\partial t}+\mathbf{B} \frac{\partial \mathbf{V}_{2}}{\partial z}=\mathbf{F}^{z}
$$

where

$$
\mathbf{V}_{2}=\left(\begin{array}{c}
u \\
w \\
q_{2}
\end{array}\right) ; \quad \mathbf{B}=\left(\begin{array}{ccc}
2 w & 0 & 0 \\
0 & 0 & 2 \\
0 & 2 & 0
\end{array}\right) ; \quad \mathbf{F}^{z}=\left(\begin{array}{c}
f_{1}^{z} \\
f_{2}^{z} \\
f_{3}^{z}
\end{array}\right)=\left(\begin{array}{c}
2 \nu \frac{\partial^{2} u}{\partial z^{2}} \\
2 \nu \frac{\partial^{2} w}{\partial z^{2}}-2 \sqrt{1-i^{2}} g \\
0
\end{array}\right)
$$

The matrix $\mathbf{B}$ has the vector of left eigenvalues $\lambda^{z}=\left(\lambda_{1}^{z}, \lambda_{2}^{z}, \lambda_{3}^{z}\right)=(2,-2,2 w)$ and the matrix of left eigenvectors

$$
\mathbf{\Omega}_{B}=\left(\begin{array}{l}
\vec{\sigma}_{1} \\
\vec{\sigma}_{2} \\
\vec{\sigma}_{3}
\end{array}\right)=\left(\begin{array}{ccc}
0 & 1 & 1 \\
0 & 1 & -1 \\
1 & 0 & 0
\end{array}\right) ; \text { and } \mathbf{\Omega}_{B}^{-1}=-\frac{1}{2}\left(\begin{array}{ccc}
0 & 0 & -2 \\
-1 & -1 & 0 \\
-1 & 1 & 0
\end{array}\right) .
$$

We obtain the complementary equations at the boundaries as follows:

- At the upper boundary:

$$
\left(\frac{d w}{d t}\right)_{z_{1}}+\left(\frac{d q_{2}}{d t}\right)_{z_{1}}=f_{2}^{z}
$$

and if $w \geq 0$ we shall have one more equation:

$$
\left(\frac{d u}{d t}\right)_{z_{3}}=f_{1}^{z}
$$


- At the lower boundary:

$$
\left(\frac{d w}{d t}\right)_{z_{3}}-\left(\frac{d q_{2}}{d t}\right)_{z_{2}}=f_{2}^{z}
$$

and if $w \leq 0$ we shall have one more equation (2.16).

\section{Numerical results of the practical problem}

We apply now the algorithm mentioned above to calculate the pressure on the vertical twodimensional valve described as follows:

$h$ is the water-depth of the sluice

$b$ is the width of the valve

$q_{\text {in }}, q_{\text {out }}$ are the input-discharge and the output-discharge

$R$ is the radius of the valve,

$A B C$ is the valve, where $A B$ is a straight line, $\mathrm{BC}$ is a part of circle with radius $R$, and $A B$ is the tangent line of $B C$ at $B$ $\mathrm{DE}$ is the free surface

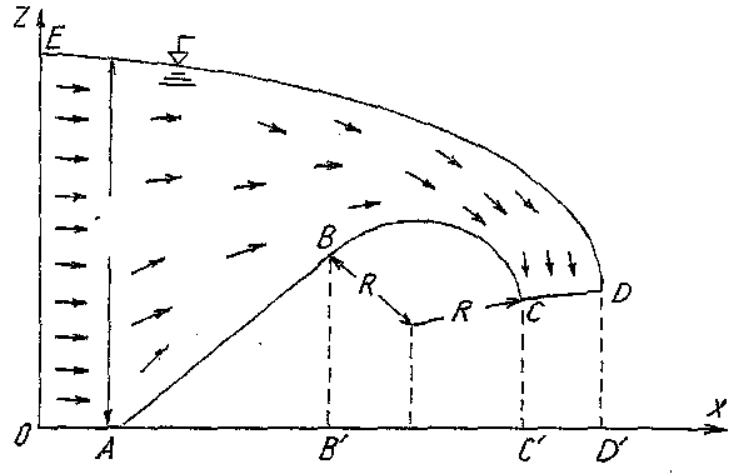

Fig. 1

We have to determine the pressure field on the valve $A B C$, with the given $E D$ (ED is the free surface). Basing on these conditions, we have steady equations:

$$
\begin{aligned}
& u \frac{\partial u}{\partial x}+w \frac{\partial u}{\partial z}+\frac{\partial p}{\partial x}=\nu \Delta u+i g \\
& u \frac{\partial w}{\partial x}+w \frac{\partial w}{\partial z}+\frac{\partial p}{\partial z}=\nu \Delta w-g \sqrt{1-i^{2}} \\
& \frac{\partial u}{\partial x}+\frac{\partial w}{\partial z}=0
\end{aligned}
$$

where $\nu=0.00001$

We add to these equations the unsteady components and we shall obtain unsteady equations like (1.3), (1.5) and apply the algorithm for interior points and boundary points mentioned above. Thus the numerical solutions are obtained. However, for these solutions and for the correctness of the problem, we have to impose the boundary conditions on each boundary as follows:

We call $u_{\vec{n}}$ as the projection of velocity vector on normal to the boundary ( $\vec{n}$ is the normal unit vector to the boundary at each boundary point directed out of the region). Then at solid boundary, we have:

$$
u_{\vec{n}}=0
$$

- At the free surface:

$$
p=0
$$

- At the soft boundary:

+ for the outflow:

$$
w=\varphi(x, z, t)
$$

or

+ for the inflow:

or

$$
\begin{aligned}
& u=\psi(x, z, t) \\
& u=\eta(x, z, t)
\end{aligned}
$$

$$
w=\varsigma(x, z, t)
$$

where $\varphi(x, z, t), \psi(x, z, t), \eta(x, z, t), \varsigma(x, z, t)$ are given values.

Thus, at each boundary sort we have 
1) At the lower boundary

a) If the boundary is solid: Consider (2.16), (2.17) and (3.1).

b) If the boundary is soft, at which water flows out $(w \leq 0)$ : Consider $(2.16),(2.17),(3.3)$ or (3.4).

2) At the upper boundary:

The boundary is the free surface: Consider (2.15), (3.1), (3.2)

3) At the left boundary:

a) If the boundary is soft, at which water flows in: Consider (2.13), (3.5) and (3.6)

b) If the boundary is solid: Consider $(2.13),(2.12)$ and $(3.1)$

4) At the right boundary:

a) If the boundary is solid: Consider (2.11), (2.12) and (3.1)

b) If the boundary is the free surface: Consider (2.11), (2.12) and (3.2)

Remark. We consider the case where the boundary is an oblique line (i.e. It is not parallel neither to $O x$ nor $O z$ ). Since our equations are splitted into two calculating process corresponding to $O x$ - and $O z$-direction, for the interior points along $O x$ direction we shall consider these oblique boundary with $O z$, and for the interior points along $O z$-direction we shall consider these oblique boundary points as boundary points being in parallel direction with $O x$. Each oblique boundary point corresponding to the above cases is arranged in the lower, upper, left and right boundary.

Applying successive iterative process to the above algorithm until at the $n^{-t h}$ step, where the values $u, w, p$ satisfy:

$$
\begin{aligned}
& \max _{m, k}\left|u_{m, k}^{n}-u_{m, k}^{n+\frac{1}{2}}\right| \leq \varepsilon\left|u_{m, k}^{n}\right| \\
& \max _{m, k}\left|w_{m, k}^{n}-w_{m, k}^{n+\frac{1}{2}}\right| \leq \varepsilon\left|w_{m, k}^{n}\right| \\
& \max _{m, k}\left|p_{m, k}^{n}-p_{m, k}^{n+\frac{1}{2}}\right| \leq \varepsilon\left|p_{m, k}^{n}\right|
\end{aligned}
$$

(where $\varepsilon$ is a precision), we shall stop and the numerical solution of the steady problem mentioned above is thus obtained.

Numerical results well conform to the experimental results carried out at Institute of Hydraulic Scientific Research in Vietnam.

The investigation shows that the numerical results are stable after 100 iterative steps and the relative error is about $0.15 \div 0.17$

This algorithm can also be applied to problem of the same models where the depth and the

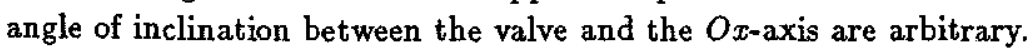

We conclude this paper with two results described in Fig. 2 and Fig. 3 respectively, and the

\begin{tabular}{|c|c|c|c|c|c|c|c|c|}
\hline \multirow{2}{*}{ Variants } & \multicolumn{8}{|c|}{ Data } \\
\hline & $\begin{array}{c}q_{\text {in }}=q_{\text {out }} \\
\left(\mathrm{m}^{3} / \mathrm{s}\right)\end{array}$ & $\begin{array}{c}h \\
(\mathrm{~m})\end{array}$ & $\begin{array}{c}b \\
(\mathrm{~m})\end{array}$ & $\begin{array}{c}R \\
(\mathrm{~m})\end{array}$ & $\begin{array}{c}\xi \\
(\mathrm{deg})\end{array}$ & $\begin{array}{l}\mathrm{AB} \\
(\mathrm{m})\end{array}$ & $\begin{array}{l}\mathrm{BC} \\
(\mathrm{m})\end{array}$ & $\begin{array}{l}\mathrm{CD} \\
(\mathrm{m})\end{array}$ \\
\hline Fig. 2 & 0.105 & 0.6 & 0.5 & 0.26 & $45^{\circ}$ & 0.32 & 0.6 & 0.1 \\
\hline Fig. 3 & 0.091044 & 0.48 & 0.5 & 0.25 & $30^{\circ}$ & 0.42 & 0.2 & 0.1 \\
\hline
\end{tabular}
data given in Table 1.

\section{Table 1}




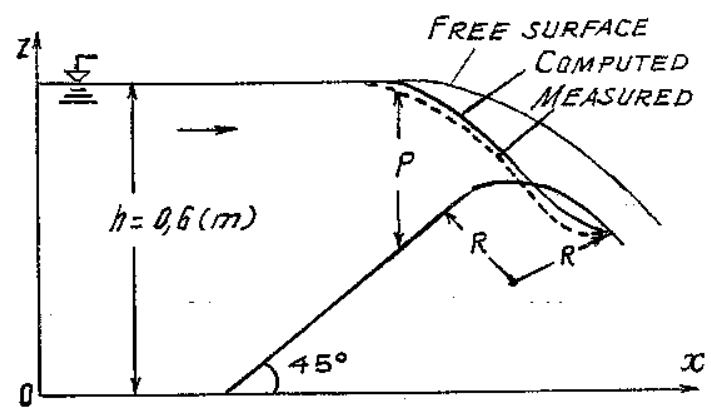

Fig. 2

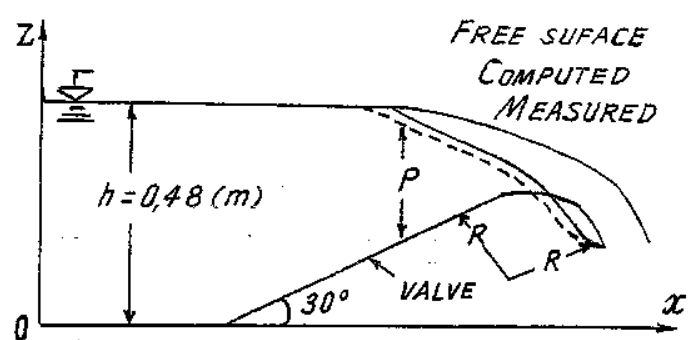

Fig. 9

Comparison computed and measured pressures on the vertical two-dimensional valve of a sluice computed

..... measured

This work is partially supported by the National Basic Research Programme for Natural Sciences and by the Programme "Applied Mathematics", NCNST.

\section{References}

1. Yanenko N. N. The method of fractional step; the solution of problems of mathematical physics in several variables (in Russian). Nauka, Novosibirk 1967.

2. Belotserkovsky O. M. Numerical modeling in mechanics of continuous media (in Russian). Nauka, Moscow 1984.

3. Tran Gia Lich, Le Viet Cuong, Nguyen Minh Son. Calculation of horizontal two-dimensional unsteady flow by the method of characteristic (in Russian). Preprint series, No 17, Hanoi 1985.

4. Nekracov V. V. Standard procedure for solving systems of quasilinear partial differential equation of hyperbolic type (in Russian). JVM \& MP No 2, 1980, 359-370.

5. Bogomolov A. L., Borokov A. I., Mairanoski F. G. Highspeed flow with free surface (in Russian). Moscow 1979.

6. Godunov S. K., Riabenki V. S. Difference schemes (in Russian). Nauka, Moscow 1973.

Received August 1, 1997

\section{TÍNH ÁP LỰC LÊN CỨ VAN}

Bài báo trình bày phương pháp số tính áp lực lên cửa van hai chiều đứng, nhờ hệ phương trình Navier-Stokes. Hệ phương trình Navier-Stokes không dù̀ng hai chiều đứng được phân rã thành hai hệ phương trình con không dù̀ng một chiều. Các tác già sử dụng sơ đồ sai phân ẫn và phương pháp truy đuổi theo từng phương để giải tù̀ng hệ phượng trình con. Tại các điểm biên nghiệm được tìm bằng phương pháp đặc trưng. 